\title{
Rigidity, locally symmetric varieties and the Grothendieck-Katz Conjecture
}

\author{
Benson Farb and Mark Kisin *
}

May 8, 2009

\begin{abstract}
Using Margulis's results on lattices in semisimple Lie groups, we prove the GrothendieckKatz $p$-Curvature Conjecture for many locally symmetric varieties, including HilbertBlumenthal modular varieties and the moduli space of abelian varieties $\mathcal{A}_{g}$ when $g>1$.
\end{abstract}

\section{Introduction}

In this paper we prove certain cases of the well-known $p$-curvature conjecture of GrothendieckKatz stated below. The conjecture posits that one can deduce algebraic solutions of certain differential equations when one has solutions after reducing modulo a prime for almost every prime. Our main purpose is to point out that rigidity theorems from the theory of discrete subgroups of Lie groups can be fruitfully applied to this problem.

More precisely, let $X$ be a smooth connected variety over $\mathbf{C}$ and let $V$ be a vector bundle on $X$ equipped with an integrable connection $\nabla$. Then there is a finitely generated $\mathbf{Z}$-algebra $R \subset \mathbf{C}$ such that $X$ arises from a smooth $R$-scheme and $(V, \nabla)$ descends to a vector bundle with integrable connection on this $R$-scheme. We will again denote by $X$ and $(V, \nabla)$ the corresponding objects over $R$.

For any maximal ideal $\mathfrak{p}$ of $R$, we can reduce $\bmod \mathfrak{p}$ to obtain a differential equation $(V / \mathfrak{p} V, \nabla)$ on a smooth scheme over a finite field of characteristic $p>0$. Attached to this system is an invariant, the $p$-curvature of $(V / \mathfrak{p} V, \nabla)$, which is an $\mathcal{O}_{X}$-linear map

$$
\psi_{p}(V, \nabla): \underline{\operatorname{Der}}\left(\mathcal{O}_{X} \otimes R / \mathfrak{p}, \mathcal{O}_{X} \otimes R / \mathfrak{p}\right) \rightarrow \underline{\operatorname{End}}_{\mathcal{O}_{X}}(V / \mathfrak{p}, V / \mathfrak{p})
$$

where Der denotes the sheaf of derivations. The map $\psi_{p}(V, \nabla)$ vanishes precisely when $(V / \mathfrak{p} V, \nabla)$ has a full set of solutions, i.e. when $V / \mathfrak{p} V$ is spanned by its subsheaf of horizontal sections.

${ }^{*}$ Both authors are supported in part by the NSF. 
Conjecture 1 (Grothendieck p-Curvature Conjecture). Suppose that for almost every maximal ideal $\mathfrak{p}$ of $R$, the $p$-curvature of $(V / \mathfrak{p} V, \nabla)$ vanishes. Then the (complex) differential equation $(V, \nabla)$ has a full set of algebraic solutions, i.e. it becomes trivial on a finite étale cover of $X$.

The truth of the conjecture does not depend on the choice of $R$ (see $\S I V$ of [Ka3]).

Katz ([Ka1], Theorem 13.0) showed that the condition on $p$-curvatures implies that $(V, \nabla)$ has regular singular points. The Riemann-Hilbert correspondence then implies that the conclusion of Conjecture 1 is equivalent to asking that the monodromy representation $\rho: \pi_{1}(X(\mathbf{C})) \rightarrow \mathrm{GL}(n, \mathbf{C})$ associated to $(V, \nabla)$ have finite image. In the same paper, Katz showed that if the $p$-curvatures vanish, and $X \hookrightarrow \bar{X}$ is a smooth compactification with $\bar{X} \backslash X$ a normal crossings divisor, then the local monodromy of $(V, \nabla)$ around a divisor at infinity is finite.

Katz also made the following more general conjecture, which describes the Lie algebra $\mathfrak{g}$ of the Tannakian Galois group $G_{\text {gal }}$ of $(V, \nabla)$. Recall that if $K(X)$ denotes the function field of the $R$-scheme $X$, then $G_{\text {gal }}$ is an algebraic group over $K(X)$, and $\mathfrak{g}$ is a Lie algebra over $K(X)$. As above one can "reduce" $\mathfrak{g}$ modulo almost any prime of $R$. We then have

Conjecture 2 (Katz). With the notation of Conjecture $1, \mathfrak{g}$ is the smallest algebraic Lie subalgebra of End $_{\mathcal{O}_{X}} V \otimes K(X)$ such that for almost every prime $\mathfrak{p}$ of $R$, the reduction of $\mathfrak{g}$ modulo $\mathfrak{p}$ contains the image of $\psi_{p}(V / \mathfrak{p} V, \nabla)$.

Let $\rho: \pi_{1}(X(\mathbf{C})) \rightarrow \mathrm{GL}_{n}(\mathbf{C})$ be the monodromy representation of $(V, \nabla)$. When $(V, \nabla)$ has regular singular points, $\mathfrak{g}$ is essentially (up to change of base ring) the Lie algebra of the Zariski closure of $\rho\left(\pi_{1}(X(\mathbf{C}))\right)$. Thus Katz's conjecture describes the topological monodromy in terms of $p$-curvatures.

Katz proved that for any $X$, Conjecture 1 is true for all $(V, \nabla)$ on $X$ if and only if Conjecture 2 is true for all $(V, \nabla)$ on $X$. For any particular $(V, \nabla)$ one knows only the obvious implication Conjecture $2 \Longrightarrow$ Conjecture 1 .

Yet another result of Katz [Ka2] says that Conjecture 1 is true when $(V, \nabla)$ is a PicardFuchs equation ${ }^{1}$ or a suitable direct factor of one. Under some conditions, this result was extended by André to $(V, \nabla)$ in the Tannakian category generated by a Picard-Fuchs equation $[\mathrm{An}, \S 16]$.

Following work of the Chudnovskys, André [An] and then Bost [Bo] proved both conjectures in the case when the monodromy representation of $\pi_{1}(X)$ has image which is virtually solvable, i.e. contains a finite index solvable subgroup. Examples of $X$ with $\pi_{1}(X)$ virtually solvable have been the only examples of varieties for which the full Grothendieck-Katz Con-

\footnotetext{
${ }^{1}$ That is, $(V, \nabla)$ arises from the de Rham cohomology of a smooth $X$-scheme.
} 
jectures (Conjectures 1 and 2) are known. In this paper we prove the conjectures for many more examples, namely for many locally symmetric varieties.

Recall that the set of complex points of a locally symmetric variety has the form $M=$ $\Gamma \backslash G(\mathbf{R})^{+} / K$, where $G$ is a connected, reductive, adjoint algebraic group over $\mathbf{Q}, G(\mathbf{R})^{+} \subset$ $G(\mathbf{R})$ denotes the connected component of the identity, $K \subset G(\mathbf{R})$ is a maximal compact subgroup of $G$ with $G(\mathbf{R}) / K$ a Hermitian symmetric domain, and $\Gamma \subset G(\mathbf{Q}) \cap G(\mathbf{R})^{+}$is an arithmetic lattice. By arithmetic lattice we mean a subgroup $\Gamma \subset G(\mathbf{Q})$ so that there exists a faithful representation $\rho: G \rightarrow \mathrm{GL}_{n}$ defined over $\mathbf{Q}$ so that $\rho(\Gamma)$ is commensurable with $\rho(G) \cap \mathrm{GL}(n, \mathbf{Z})$. Note that since $G$ is an adjoint group, we have $G=G_{1} \times \cdots \times G_{m}$ with each $G_{i}$ adjoint and $\mathbf{Q}$-simple.

Our first result proves the Grothendieck-Katz Conjectures for all "higher rank" locally symmetric varieties $M$ for which $G$ has no $\mathbf{Q}$-simple factors of exceptional type.

Theorem 3. Let $M$ be a locally symmetric variety, as above, and suppose that each factor $G_{i}$ has $\mathbf{R}$-rank $\geq 2$ and is of type $A, B, C$, or D. Then the Grothendieck-Katz conjecture holds for any $(V, \nabla)$ over $M$.

Examples to which Theorem 3 applies include the Hilbert-Blumenthal modular varieties, as well as the moduli space $\mathcal{A}_{g}, g>1$ of principally polarized abelian varieties. If the factors $G_{i}$ all have $\mathbf{Q}$-rank $\geq 1$, we give a different proof of the theorem, which is also valid for groups of exceptional type.

Theorem 4. Let $M$ be a locally symmetric variety, as above, and suppose that each factor $G_{i}$ has $\mathbf{R}$-rank $\geq 2$ and $\mathbf{Q}-r a n k \geq 1$. Then the Grothendieck-Katz conjecture holds for any $(V, \nabla)$ over $M$.

Note that Theorem 3 and Theorem 4 omit the case when $M$ is a curve, a case which would imply (by standard methods) the full Conjecture 2. This is no accident; our method applies rigidity results which are false in the case of curves.

The proof of both theorems procedes by first deducing a result on the structure of vector bundles on locally symmetric varieties from one of Margulis' rigidity results. This is then combined with results of Katz and André mentioned above. The proof of Theorem 3 combines Andre's extension of Katz's solution to Conjecture 2 for Picard-Fuchs equations with the Margulis Superrigidity Theorem. The proof of Theorem 4 combines Katz's result on local monodromy with the Margulis Normal Subgroup Theorem. Thus we have two combinations of two quite different pairs of theorems combining to give similar results.

Locally symmetric varieties are naturally equipped with a collection of variations of Hodge structure (see $\S 3$ below for a more precise discussion). The underlying vector bundles with connection of these variations of Hodge structure form an interesting class of examples 
to which Theorem 3 may be applied. In this case the Lie algebra $\mathfrak{g}$ in Conjecture 2 is a factor of Lie $G$. We refer to these bundles as automorphic bundles. In fact we show that in the situation of Theorem 3 , essentially any $(V, \nabla)$ on $M$ with regular singular points is a direct summand of an automorphic bundle.

Acknowledgements. We are grateful to Sasha Beilinson for useful discussions, and to the referee for several useful comments.

\section{Proof of Theorem 4}

\section{$2.1 \quad$ Toroidal compactifications}

As above, let $G$ be a connected, reductive, adjoint group over $\mathbf{Q}$, and let $K \subset G(\mathbf{R})^{+}$be a maximal compact subgroup such that $D=G(\mathbf{R})^{+} / K$ is a Hermitian symmetric domain. Let $\Gamma$ be a torsion-free arithmetic subgroup in $G(\mathbf{R})^{+} \cap G(\mathbf{Q})$, and let $M=\Gamma \backslash G(\mathbf{R})^{+} / K$ be the corresponding locally symmetric variety.

We recall the theory of toroidal compactifications for locally symmetric varieties [AMRT]. Such a compactification $\bar{M}^{\text {tor }} \supset M$ is a projective variety admitting $M$ as a dense open subset. The identity map extends to a (unique) continuous map from $\bar{M}^{\text {tor }}$ to the BailyBorel compactification. By construction the boundary $\bar{M}^{t o r} \backslash M$ is a union of non-empty closed subsets indexed by maximal parabolic subgroups $G$ which are defined over $\mathbf{Q}$.

The toroidal compactification is not unique; but depends on a choice of combinatorial data. This data can always be chosen so that $\bar{M}^{\text {tor }}$ is smooth and $\bar{M}^{\text {tor }} \backslash M$ is a divisor with normal crossings in $\bar{M}^{\text {tor }}$. From now on we fix a compactification with this property. A loop in $M$ around an irreducible component of $\bar{M}^{\text {tor }} \backslash M$ corresponds to a non-trivial unipotent element in $\Gamma$ (see $[$ AMRT], III, §5, Main Thm. I).

Proposition 5. Suppose that $G$ is $\mathbf{Q}$-simple with $\mathbf{R}$-rank $\geq 2$ and $\mathbf{Q}$-rank $\geq 1$. Let $(V, \nabla)$ be a vector bundle with integrable connection on $M$. If $(V, \nabla)$ has finite local monodromy around the components of $\bar{M}^{\text {tor }} \backslash M \subset \bar{M}^{\text {tor }}$, then the monodromy representation $\rho$ of $(V, \nabla)$ has finite image.

Proof. Since the $\mathbf{Q}$-rank of $G$ is $\geq 1, G$ has a parabolic subgroup defined over $\mathbf{Q}$ and the boundary is nonempty. Let $\gamma \in \Gamma$ be an element represented by a loop around an irreducible component of $\bar{M}^{\text {tor }} \backslash M$. Then $\gamma$ is a nontrivial unipotent element, and in particular has infinite order. Since $\rho(\gamma)$ is finite, by assumption, the kernel of $\rho$ is infinite.

Now since the Q-rank of $G$ is $\geq 1, G(\mathbf{R})$ has no compact factors. Since $G$ is $\mathbf{Q}$-simple, $\Gamma \subset G(\mathbf{R})$ is an irreducible lattice in a real semisimple Lie group of real rank at least 2. These properties of $G$ and $\Gamma$ imply that we can apply the Margulis Normal Subgroup 
Theorem ([Ma], IX, 6.14), which states that any normal subgroup of such a lattice is finite and central or has finite index. Since $\operatorname{ker}(\rho) \triangleleft \Gamma$ is infinite, it follows that $\operatorname{ker}(\rho)$ has finite index in $\Gamma$, i.e. that $\rho(\Gamma)$ is finite, and we are done.

\subsection{Proof of Theorem 4}

First we may replace $\Gamma$ by a finite index subgroup, and assume that $\Gamma$ has the form $\Gamma_{1} \times$ $\cdots \times \Gamma_{m}$ where $\Gamma_{i} \subset G_{i}(\mathbf{Q}) \cap G_{i}(\mathbf{R})^{+}$is an arithmetic subgroup. To prove the theorem it suffices to prove the analogous result for each $G_{i}$. Replacing $G$ by $G_{i}$, we may assume that $G$ is $\mathbf{Q}$-simple.

By the result of Katz ([Ka3], Thm. 10.2) already mentioned above, it suffices to prove Conjecture 1 for an arbitrary vector bundle with integrable connection $(V, \nabla)$ on $M$. Thus we may assume that the $p$-curvatures of $(V, \nabla)$ vanish for almost all $\mathfrak{p}$.

According to [Ka1], Thm. 13.0, since the $p$-curvatures of $(V, \nabla)$ are almost all 0 , the local monodromy groups of $(V, \nabla)$ around the components of $\bar{M}^{\text {tor }} \backslash M$ are finite groups, so $\rho(\gamma)$ is finite by Proposition 5 .

\section{Automorphic bundles and superrigidity}

\subsection{Superrigidity}

We will need the following theorem, which is part (iii) of Theorem (B) in Chapter VIII of [Ma], stated with $K=\mathbf{Q}, \ell=\mathbf{R}$, and with $S$ containing only archimedean places.

Theorem 6 (Margulis). Let $G$ be a connected, simply connected, semisimple, almost $\mathbf{Q}$ simple algebraic Q-group, and let $\Gamma$ be an arithmetic lattice in $G$. Let $H$ be an algebraic $\mathbf{R}$-group, and let $\psi: \Gamma \rightarrow H$ be a homomorphism. Assume that $\operatorname{rank}_{\mathbf{R}}(G) \geq 2$. Then there exists an $\mathbf{R}$-morphism $\phi: G \rightarrow H$ and a homomorphism $\nu: \Gamma \rightarrow H$ so that $\nu(\Gamma)$ is finite and

$$
\psi(g)=\nu(g) \phi((g)) \quad \text { for all } g \in \Gamma
$$

Theorem 6 is a form of the Margulis Superrigidity Theorem. This theorem is often stated in the form "either $\psi$ induces a map $\phi: G \rightarrow H$ or $\psi(\Gamma)$ has compact closure." However in the case of arithmetic lattices one has the more precise statement given above.

\subsection{Automorphic bundles}

Let $G, M$ and $\Gamma$ be as in the introduction, and let $\tilde{G}$ be a connected reductive group over $\mathbf{Q}$ with $\tilde{G}^{\text {ad }}=G$. Recall that there is a unique $G(\mathbf{R})$ conjugacy class of cocharacters 
$h: \mathbf{C}^{\times} \rightarrow G(\mathbf{R})$ such that the centralizer of $h$ in $G(\mathbf{R})$ is compact. Fix such a cocharacter, and suppose that $h$ lifts to $\tilde{h}: \mathbf{C}^{\times} \rightarrow \tilde{G}(\mathbf{R})$.

Attached to any representation of $\tilde{G}$ (defined over $\mathbf{Q}$ ) there is a canonical variation of Q-Hodge structure on $M$ [De, 1.1.12]. In particular, a representation of $\tilde{G}$ gives rise to an algebraic vector bundle on $M$ equipped with an integrable connection, having regular singular points. We call a vector bundle with connection arising in this way an automorphic bundle.

For example, if $M$ is (a component of) a moduli space of principally polarized abelian varieties, and $\tilde{G}$ is a general symplectic group equipped with its standard representation, then the corresponding automorphic bundle arises from degree 1 cohomology of the universal family of abelian varieties over $M$.

Theorem 7. Let $G$ be $\mathbf{Q}$-simple with $\mathbf{R}$-rank $\geq 2$, and let $(V, \nabla)$ be a vector bundle with connection on $M$ having regular singular points. Then there exists a finite covering $\tilde{M} \rightarrow M$ such that the restriction of $(V, \nabla)$ to $\tilde{M}$ is a direct summand of an automorphic bundle.

Proof. We will write $G_{\mathbf{R}}$ for $G$ viewed as an algebraic group over $\mathbf{R}$. Since $(V, \nabla)$ has regular singular points it suffices to show that its associated monodromy representation is a direct summand of one attached to an automorphic bundle. Let $\rho: \Gamma \rightarrow \operatorname{GL}_{n}(\mathbf{C})$ denote the monodromy representation of $(V, \nabla)$. Viewing $\mathbf{C}$ as an $\mathbf{R}$-vector space, and replacing $n$ by $2 n$, we may assume that $\rho$ factors through $\mathrm{GL}_{n}(\mathbf{R})$.

Now let $\tilde{G}^{\text {der }}$ denote the universal cover of $G$. After replacing $\Gamma$ by a finite index subgroup, we may view $\Gamma$ as a subgroup of $\tilde{G}^{\text {der }}$. Moreover, we may assume that $\rho$ factors through $\mathrm{SL}_{n}(\mathbf{R})$. By Theorem 6, after again replacing $\Gamma$ by a finite index subgroup on which $\nu$ is trivial, we have that $\rho_{\text {alg }}$ is induced by a map of algebraic $\mathbf{R}$-groups $\rho_{\text {alg }}: \tilde{G}_{\mathbf{R}}^{\text {der }} \rightarrow \mathrm{SL}_{n}$.

Let $Z=\operatorname{ker}\left(\tilde{G}^{\text {der }} \rightarrow G\right)$ be the center of $\tilde{G}^{\text {der }}$. Choose a Q-torus $T$, and an embedding of $\mathbf{Q}$-groups $Z \hookrightarrow T$. Let $K / \mathbf{Q}$ be a quadratic imaginary extension, and set

$$
\tilde{G}=\tilde{G}^{\text {der }} \times{ }_{Z} R_{K / \mathbf{Q}} T .
$$

where $R_{K / \mathbf{Q}}$ denotes restriction of scalars from $K$ to $\mathbf{Q}$. Then $h: R_{\mathbf{C} / \mathbf{R}} \mathbf{G}_{m} \rightarrow G_{\mathbf{R}}$ lifts to $\tilde{G}$. Fix such a lifting.

Let $\tilde{G} \hookrightarrow \mathrm{GL}\left(W_{\mathbf{Q}}\right)$ be a faithful representation on a $\mathbf{Q}$-vector space $W_{\mathbf{Q}}$. Then $W_{\mathbf{R}}=$ $W_{\mathbf{Q}} \otimes_{\mathbf{Q}} \mathbf{R}$ is a faithful representation of $\tilde{G}_{\mathbf{R}}^{\text {der }}$, so $\rho_{\text {alg }}$ is a direct summand of a $\tilde{G}_{\mathbf{R}}^{\text {der }}$ representation of the form $W_{\mathbf{R}}^{\otimes n} \otimes W_{\mathbf{R}}^{* \otimes m}$, where $W_{\mathbf{R}}^{*}$ denotes the dual of $W_{\mathbf{R}}$. Then the composite

$$
\Gamma \hookrightarrow \tilde{G}^{\mathrm{der}}(\mathbf{R}) \stackrel{\rho_{\text {alg }}}{\rightarrow} \mathrm{SL}_{n}(\mathbf{R}) .
$$

is a direct summand of $W_{\mathbf{R}}^{\otimes n} \otimes W_{\mathbf{R}}^{* \otimes m}$, whose complexification is a representation associated to an automorphic bundle. 


\subsection{Proof of Theorem 3}

André's Theorem says that Conjecture 2 holds when $(V, \nabla)$ is in the Tannakian category generated by a Picard-Fuchs equation attached to a smooth projective morphism $f: Y \rightarrow X$, provided one of the fibres $Y_{x}$ of $f$ has a connected motivic Galois group. André defines this group using his theory of motivated cycles. Here we remark only that when $f$ is a family of abelian varieties, the motivic Galois group is equal to the Mumford-Tate group of $Y_{x}$, and hence is connected [An, 16.2, 16.3].

As in the proof of Theorem 4, we may assume that $G$ is $\mathbf{Q}$-simple. Let $\tilde{G}^{\text {der }}$ be as in the proof of Theorem 4 . Since we may replace $\Gamma$ by a finite index subgroup we may assume that $\Gamma \subset \tilde{G}^{\text {der }}(\mathbf{R})$, as before.

Let $(V, \nabla)$ on $M$ have vanishing $p$-curvatures for almost every $\mathfrak{p}$, and $\rho$ the corresponding representation of $\Gamma$. As above, we may assume that $\rho$ is induced by a representation $\rho_{\text {alg }}$ of the real algebraic group $\tilde{G}^{\text {der }}$. Let $V_{\mathbf{C}}$ denote the underlying complex vector space of $\rho_{\text {alg }}$, and decompose $V_{\mathbf{C}}$ as a sum of representations on each of which the center $Z \subset \tilde{G}^{\text {der }}$ acts via a character. It suffices to consider each summand individually, so we may assume that $\rho(Z(\mathbf{C}))$ consists of scalars.

For any $g \geq 1$, let $\mathcal{H}_{g}$ denote $\operatorname{Sp}_{2 g}(\mathbf{R})$ modulo a maximal compact subgroup. The condition on the type of the $\mathbf{Q}$-simple factors of $G$ implies ([De] $\S 1.3$ ) that for some $g \geq 1$, there exists an arithmetic subgroup $\Gamma_{g} \subset \operatorname{Sp}_{2 g}(\mathbf{R})$, a finite index subgroup $\Gamma^{\prime} \subset \Gamma$, and a map of complex varieties

$$
\Gamma^{\prime} \backslash G(\mathbf{R}) / K \rightarrow \Gamma_{g} \backslash \mathcal{H}_{g}
$$

such that the corresponding map on fundamental groups $\Gamma^{\prime} \rightarrow \Gamma_{g}$ is induced by a map of Q-groups $\tilde{G}^{\text {der }} \rightarrow \mathrm{Sp}_{2 g}$ with finite central kernel.

Let $W_{\mathbf{Q}}$ denote the representation of $\tilde{G}^{\text {der }}$ which is induced by the standard representation of $\mathrm{Sp}_{2 g}$. Then $W_{\mathbf{Q}}$ is attached to an automorphic bundle $\left(W, \nabla_{W}\right)$ which arises as the cohomology of a family of abelian varieties on $M$. Hence by André's result, mentioned above, the Grothendieck-Katz conjecture holds for all bundles in the Tannakian category generated by $\left(W, \nabla_{W}\right)$.

Now the bundle (End $V, \nabla_{\text {End } V}$ ) has regular singular points, and the corresponding monodromy representation $\operatorname{End} \rho$ factors through $G$, as $Z$ acts on $V_{\mathbf{C}}$ via scalars. Hence End $V$ is in the Tannakian category generated by $\left(W, \nabla_{W}\right)$. It follows that $(\operatorname{End} \rho)(\Gamma)$ is a finite group, and replacing $\Gamma$ by a finite index subgroup we may assume it is trivial.

It follows that $\Gamma$ acts on $V_{\mathbf{C}}$ by scalars, and hence so does $\tilde{G}^{\text {der }}$, as $\Gamma$ is Zariski dense in $\tilde{G}^{\text {der }}$. As the latter group is connected and semisimple, we conclude that $\Gamma$ acts trivially on $V_{\text {C. }}$ 


\section{References}

[AMRT] A. Ash, D. Mumford, M. Rapoport, Y. Tai, Smooth compactifications of locally symmetric varieties, Lie Groups: History, frontiers and applications IV (1975).

[An] Y. André, Sur la conjecture des $p$-courbures de Grothendieck-Katz et un problème de Dwork, Geometric aspects of Dwork theory, 55-112, (2004).

[Bo] J.B. Bost, Algebraic leaves of algebraic foliations over number fields, Publ. Math. IHES, Vol. 93 (2001), 161-221.

[De] P. Delgine, Variétés de Shimura: interprétation modulaire et techniques de construction de modèles canoniques, Automorphic forms, representations and L-functions (Corvallis 1977), Proc. Sym. Pure Math. 33 (1979), 247-290.

[Ka1] N. Katz, Nilpotent connections and the monodromy theorem: Applications of a result of Turrittin, Publ. Math IHES, No. 39 (1970), 175-232.

[Ka2] N. Katz, Algebraic solutions of differential equations ( $p$-curvature and the Hodge filtration), Invent. Math. 18 (1972), 1-118.

[Ka3] N. Katz, A conjecture in the arithmetic theory of differential equations, Bull. SMF 110 (1982), 203-239.

[Ma] G. Margulis, Discrete subgroups of semisimple Lie groups, Ergebnisse der Math. 17 (1991).

[Wi] D. Witte Morris, Introduction to Arithmetic Groups, preliminary version, 2008.

Dept. of Mathematics, University of Chicago

5734 University Ave.

Chicago, Il 60637 